\title{
Electronic and Thermal Properties of Biphenyl Molecules
}

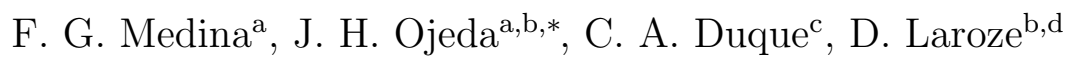 \\ ${ }^{a}$ Grupo de Física de Materiales, Universidad Pedagógica y Tecnológica de Colombia, Tunja, Colombia \\ ${ }^{b}$ Instituto de Alta investigación, Universidad de Tarapacá, Casilla $7 D$ Arica, Chile \\ ${ }^{c}$ Grupo de Materia Condensada-UdeA, Instituto de Física, Facultad de Ciencias Exactas Naturales, Universidad de \\ Antioquia UdeA, Calle 70 No. 52-21, Medellín, Colombia \\ ${ }^{d}$ SUPA School of Physics and Astronomy, University of Glasgow, Glasgow G12 8QQ, United Kingdom
}

\begin{abstract}
Transport properties of a single Biphenyl molecule coupled to two contacts are studied. We characterise this system by a tight-binding Hamiltonian. Based on the non-equilibrium Green's functions technique with a Landauer-Büttiker formalism the transmission probability, current and thermoelectrical power are obtained. We show that the Biphenyl molecule may have semiconductor behaviour for certain values of the electrode-molecule-electrode junctions and different values of the angle between the two rings of the molecule. In addition, the density of states (DOS) is calculated to compare the bandwidths with the profile of the transmission probability. DOS allows us to explain the asymmetric shape with respect to the molecule's Fermi energy.
\end{abstract}

Keywords: Thermopower, Density of states, Green's functions.

\section{Introduction}

The molecular electronics has had a remarkable progress during the past decades, in particular by the synthesis of molecules and assembling them to electronic devices, getting new applications in nanoscale systems. The transport properties have been determined, in order to use molecular systems in practical devices such as in organic solar cells, organic light-emitting diodes, molecular circuits, and organic field-effect transistors, just to mention a few [1-4].

These systems may be based on single-molecules and have been studied from both, the theoretical and experimental point of view. In fact, it has been found that the mechanisms of different electronic behaviours depends on the molecule's geometry or the external agents [5-8]. The deep understanding of the transport and thermal prop-

\footnotetext{
*Corresponding authors

Email address: judith.ojeda@uptc.edu.co (J. H. Ojeda )
}

erties of aromatic molecules helps us in the development of molecular devices, and for that reason we focus in the present work on the study of the electronic as well as thermodynamic properties of the Biphenyl molecule $\left(C_{12} H_{8}\right)$ coupled to two semi-infinite leads.

The aim of this work is to explore both, the electrical and the thermal fluctuations, in the transport through the Biphenyl molecule. In particular, we study these properties by changing the geometry of the molecule and by assuming different regimes of the coupling between the molecule and the contacts. The molecule is modeled by a tightbinding Hamiltonian with a nearest-neighbour approximation. The calculations of the transport properties based on electron transfer and heat transport are performed using non-equilibrium Green's functions techniques inside of a discrete space. In particular, we use a decimation procedure or a real-space renormalisation scheme [9-11]. The transmission probability, thermoelectrical power and current as a function of the bias voltages are 
obtained following the Landauer-Büttiker formalism [12]. In addition, we calculate the density of states (DOS) to compare the bandwidths with the profile of the transmission probability and the Seebeck coefficient in the limit of strong and weak couplings. We show a good agreement between both methods.

The manuscript is organised as follows: In Section 2, the theoretical model is introduced and the system's transport quantifiers are described. In Section 3 numerical simulations are performed and the results are explained. Finally, a summary is given in Section 4.

\section{Theoretical Model}

To describe the Biphenyl aromatic molecule as an electronic device, the molecule is connected to contacts at both ends. Each ring consists of $n=6$ carbon atoms. The set-up is depicted in Figure 1.



Figure 1: (Color online) Biphenyl aromatic molecule.

The Hamiltonian of the total system in the a tight-binding approximation is given by:

$$
\hat{H}=\hat{H}_{m o l}+\hat{H}_{e}+\hat{H}_{c},
$$

where $\hat{H}_{m o l}$ is the Hamiltonian of the Biphenyl molecule without contacts which can be written as:

$$
\begin{aligned}
\hat{H}_{m o l}= & \sum_{i} \epsilon \hat{c}_{i}^{\dagger} \hat{c}_{i}+\sum_{i} v\left[\hat{c}_{i+1}^{\dagger} \hat{c}_{i}+\hat{c}_{i}^{\dagger} \hat{c}_{i+1}\right] \\
& +\sum_{j} \epsilon \hat{c}_{j}^{\dagger} \hat{c}_{j}+\sum_{j} v\left[\hat{c}_{j+1}^{\dagger} \hat{c}_{j}+\hat{c}_{j}^{\dagger} \hat{c}_{j+1}\right] \\
& +t\left[\hat{c}_{4}^{\dagger} \hat{c}_{7}+\hat{c}_{7}^{\dagger} \hat{c}_{4}\right] .
\end{aligned}
$$

Here, $\epsilon$ is the electron energy per site or per carbon atom, $c_{i}^{\dagger}$ is the creation operator of an electron at site $i$ in the ring $1, c_{j}^{\dagger}$ is the creation operator of an electron at site $j$ in the ring $2, v$ is the hopping parameter between carbon atoms and $t$ is the torsional potential. The Hamiltonian which represents the electrodes, $\hat{H}_{e}$, is given by:

$$
\hat{H}_{e}=\sum_{k_{R}} \epsilon_{k_{R}} \hat{d}_{k_{R}}^{\dagger} \hat{d}_{k_{R}}+\sum_{k_{L}} \epsilon_{k_{L}} \hat{d}_{k_{L}}^{\dagger} \hat{d}_{k_{L}}
$$

Finally, the Hamiltonian that describes the interaction between the contacts and molecule can be cast in the form:

$$
\hat{H}_{c}=\sum_{k_{R}} \Gamma_{R} \hat{d}_{k_{R}}^{\dagger} \hat{c}_{1}+\sum_{n_{L}} \Gamma_{L} \hat{d}_{k_{L}}^{\dagger} \hat{c}_{N}+\text { h.c. },
$$

where the operator $\hat{d}_{k_{L(R)}}^{\dagger}$ is the creation operator of an electron in a state $k_{L(R)}$ with energy $\epsilon_{k_{L(R)}}$, while $\Gamma_{R(L)}$ is the coupling energy between the right (left) lead with the Biphenyl molecule.

The thermodynamic and electronic properties of a single Biphenyl molecule coupled to two contacts are calculated through the Landauer-Büttiker formalism [12] based on the Green's function techniques. In particular, the Green's functions are computed within a real-space renormalisation approach. This method allows us to obtain all the electronic information of the molecular system [13]. In this scenario, the resultant molecular system is reduced to an unidimensional system with effective energy sites and effective couplings between them as is shown in Figure 2.

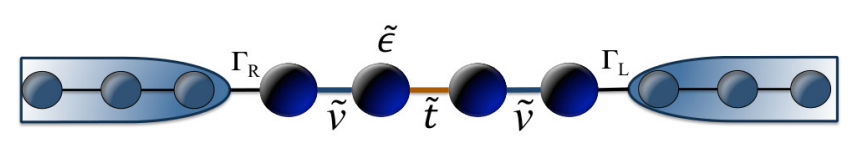

Figure 2: (Color online) Effective Biphenyl aromatic molecule.

The Green's function of the aromatic molecule coupled to the leads is calculated using the Dyson equation, which is given by

$$
G=G_{0}+G\left(\Sigma_{L}+\Sigma_{R}\right) G_{0},
$$

where $G^{0}$ is the bare Green's function of the isolated aromatic molecule and $\Sigma_{L}$ and $\Sigma_{R}$ are the 
self-energies of the left and right lead, respectively. The detail of the renormalization method and analytical expressions of the Green's functions are given in the Appendix. The transmission probability in the reduced system can be calculated within the Fischer-Lee relationship [14]:

$$
T(E)=\Gamma_{11}^{L} \Gamma_{N N}^{R}\left|G_{1 N}^{r}\right|^{2},
$$

where $\Gamma_{11}^{L}$ and $\Gamma_{N N}^{R}$ depict the element $\{1,1\}$ and $\{N, N\}$ of the effective spectral matrix density of the left and right lead, respectively. The current passing through the aromatic molecule can be considered as a scattering process of an electron between the leads. Using the Landauer formalism, the $I-V$ characteristics can be obtained by the following expression

$$
I(V)=I_{0} \int_{-\infty}^{\infty}\left(f_{L}-f_{R}\right) T(E) d E
$$

where $I_{0}=2 e / h$ and $f_{L(R)}$ is the Fermi-Dirac distribution function evaluated at $f_{L(R)}=f(E-$ $\left.\mu_{L(R)}\right)$, where $\mu_{L(R)}=E_{f} \pm \mathrm{eV} / 2$ is the chemical potential. Here $e$ is the electron charge and $h$ is the Planck constant. Based on the same formalism, it is possible to find an expression for the thermopower of a lead-molecule-lead junction. It is called Seebeck coefficient, $(Q)$, which in terms of the transmission probability can be written as [15-17, 23]:

$$
Q=-\left.Q_{0} \frac{\partial \ln T(E)}{\partial E}\right|_{E=E_{f}}
$$

where $Q_{0}=\pi^{2} k_{B}^{2} \Theta /(3|e|)$, with $k_{B}$ the Boltzmann constant and $\Theta$ the temperature. Finally, we remark that one can calculate the DOS using the Green's function through the expression $D O S=\frac{i}{2 \pi} \operatorname{Tr}\left[G_{j j}-G_{j j}^{\dagger}\right]$.

\section{Results and Discussions}

In this section we analyze electric and thermal properties of the biphenyl molecule depending on the energy, the lead-molecule-lead coupling $(\Gamma)$ and the variation of the angle between the rings, which is given by $t=v \cos \varphi$ [18]. We focus on two coupling regimes, the weak coupling, where $\Gamma$ is smaller than the inter-site coupling $v(\Gamma<v)$, and the strong coupling regime with $\Gamma \sim v$.
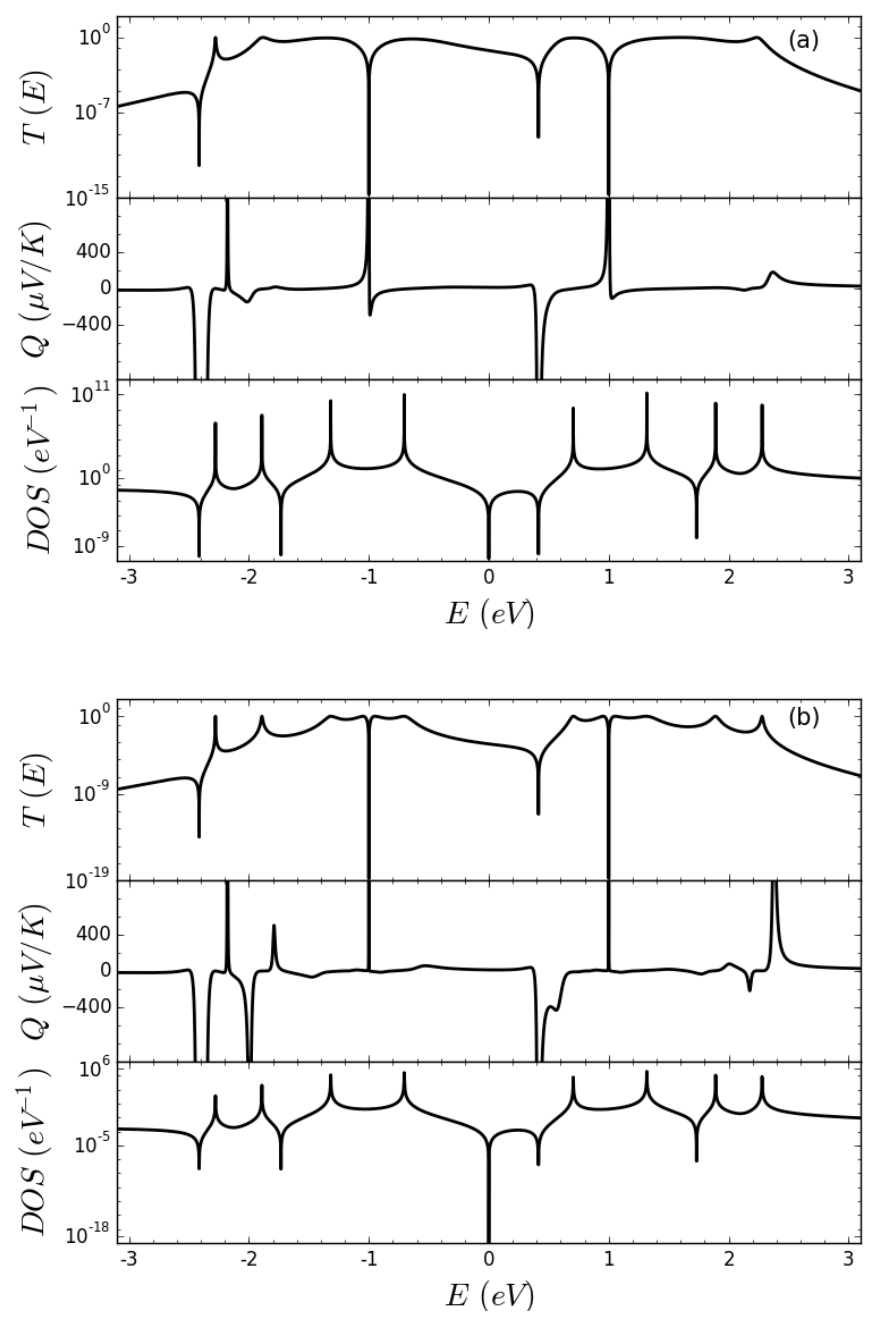

Figure 3: Transmission probability $(T)$, Seebeck Coefficient $(Q)$ and DOS as a function of the energy $E$. For upper and lower panels the scale is worked in log, at $\Gamma=1.0 \mathrm{eV}$ (a) and $\Gamma=0.1 \mathrm{eV}$ (b). The fixed parameters are $E_{f}=0 \mathrm{eV}, v=1.0 \mathrm{eV}$ and $\Theta=120 \mathrm{~K}$.

The results for the transmission probability, the Seebeck coefficient, and the DOS as a function of energy for the biphenyl molecule are presented in Figure 3. The panel (a) displays a strong coupling regime, while the panel (b) a weak one. The values of the coupling constant are $\Gamma=1.0 \mathrm{eV}$ and $\Gamma=0.1 \mathrm{eV}$, respectively. In panel (a) one can observe that the transmission probability shows resonant tunnelling, since there are multiple uni- 
tary resonant peaks, which are associated with the eigenvalues of each atomic site of molecule around of the equilibrium Fermi energy $E_{f}=0$. It is worth mention that there is quantum null interference phenomenon, which in this case is given at energies -1 and 1 . The antiresonance arises from the destructive interference between the delocalized states of the contact and the localized states in each atom when the electron cross the interface between them. This issue is in agreement with recent works in other molecular systems [19-22].

If we compare the transmission and Seebeck spectra, we notice, for energies where the transmission is zero, the Seebeck coefficient has a positive or negative peak. Generally, when the Seebeck coefficient is positive (negative), it represents a p-type (n-type) semiconductor behavior. Our results for the transmission and the Seebeck coefficient are in agreement with the previous results on Benzene, reported in Refs. [10, 19, 23].

For the weak regime one can observe some differences in the transmission probability resonances. They have opened the wide band as it is shown in the panel (b). This phenomenon is due to the hybridization of energy levels produced by the contacts on the molecule [24-26]. For the Seebeck coefficient we observe that this curve is smoothed due to the expansion of the resonances in the transmission. On the other hand, the values of the DOS increase slightly in the strong regime compared to the weak one. We remark that the width of the DOS peaks match the width of the transmission peaks.

Note that the transmission and DOS present a peculiar asymmetry in height of the peaks around the Fermi energy, where the height in HOMO peak is larger than the corresponding one of the LUMO peak - this feature is accentuated in strong coupling regime. This behaviour is due to the fact that the LUMO level in the molecule is closer to electrodes than the HOMO level. When the system has a weak coupling, it is similar to have the electrodes away to the system, therefore the DOS decrease and the transmission spectra try to keep the symmetry in the width bands. When the contacts are near to the system, the coupling becomes strong and the DOS increase considerably.
In order to support the above explanation, Figure 4 shows the DOS as a function of the energy for the strong coupling in its whole range. Now, it is more evident that the distribution of the DOS around $E_{f}=0$ is asymmetric. Also, we can observe on the side of low-unoccupiedmolecular-orbital (LUMO) a greater amount of DOS compared to a high-occupied-molecular-orbital (HOMO). The corresponding energies for these two states are approximately close to $0.5 \mathrm{eV}$ and $-0.5 \mathrm{eV}$, respectively.

The zoom into the Figure 4 shows more clearly the number of occupied and empty states, where the number of occupied states match with the number of peaks in the transmission spectra for strong coupling.



Figure 4: DOS as a function of the energy, E. The zoom shows DOS with a lower scale. The fixed parameters are $\epsilon=0, v=1 \mathrm{eV}$ and $\Gamma=1.0 \mathrm{eV}$.

Figure 5 represents the Seebeck coefficient as function of temperature and energy for strong (Fig. 5a) and weak (Fig. 5b) couplings. The dotted lines represent the temperature at which the Seebeck coefficient was obtained in (fig. 3a) and (fig. $3 \mathrm{~b}$ ), respectively. In the strong regime we can observe at $300 K$ a better conduction of the holes (at $-1 \mathrm{eV}$ ) and of the electrons (at $1 \mathrm{eV}$ ). In the weak regime the values of the thermopower are lower than the corresponding values for strong 


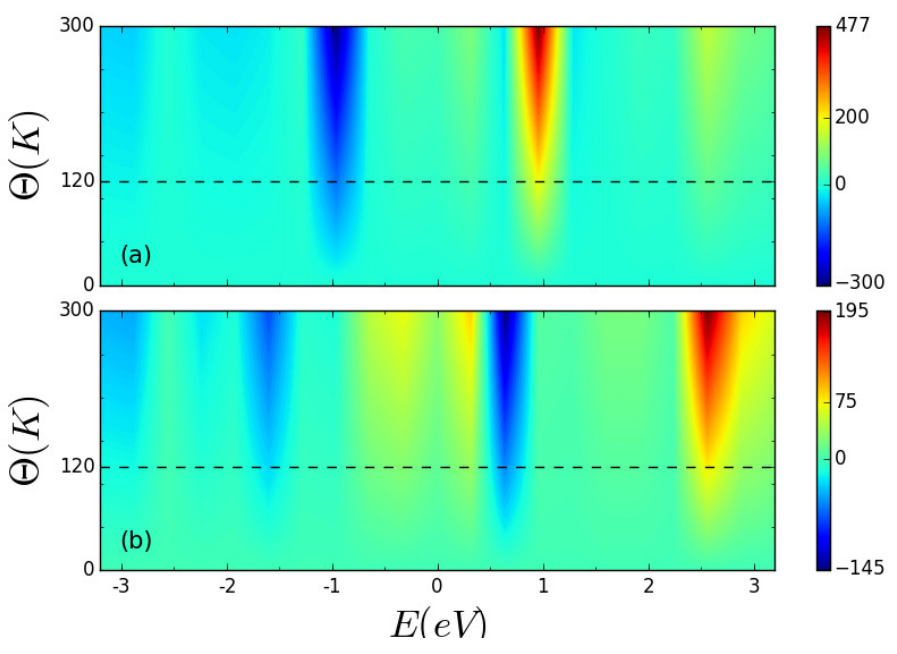

Figure 5: (Color online) Contour plot of the Seebeck coefficient as a function of temperature $(\Theta)$ and energy, $E$. (a) Strong coupling and (b) Weak coupling. The rest of the fixed parameters are the same of Fig. 3.

coupling, but more resonances are presented at different values of the energy. For instance, at $2.5 \mathrm{eV}$ conduction of electrons appears in a range of $120 \mathrm{~K}$ to $300 \mathrm{~K}$. Note that for energies between HOMO and LUMO, the conduction of the holes is predominant.

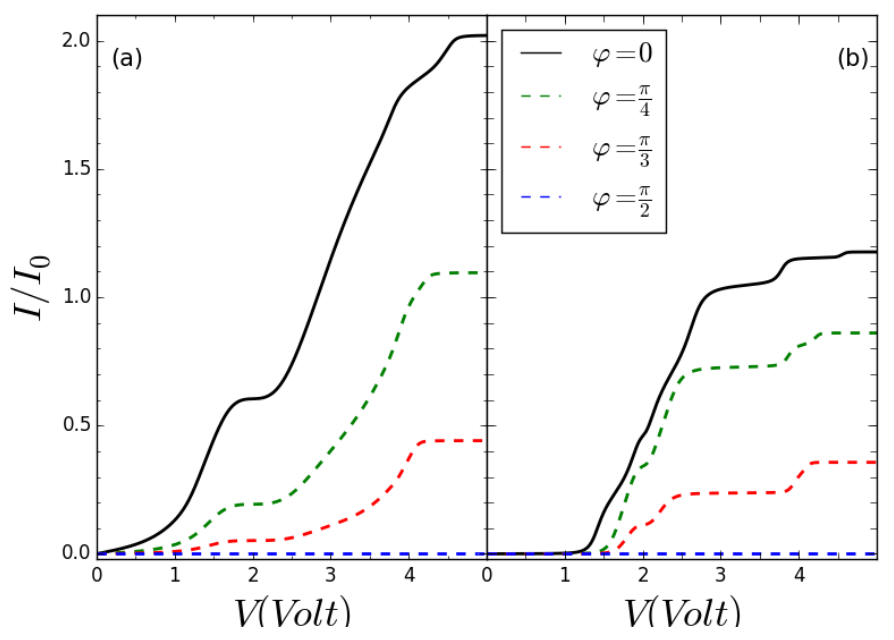

Figure 6: (Color online) Normalised current $I / I_{0}$ as a function of the voltage $V$ for different aromatic torsion angles. The frames (a) and (b) are for strong and weak coupling respectively. The other fixed parameters are the same of Fig. 3.

Figure 6 shows the dependence of the current on the voltage for both coupling regimes. We can observe that for strong coupling the amplitudes of the current are greater than for weak coupling. This effect is due to the hybridization of molecular orbitals due to the contacts. Also, we observe that the width of the gap voltage decreases when the strength of coupling increases. In addition, when the aromatic torsion angle is $\pi / 2$, the current vanishes. Finally, let us remark that for both regimes the number of steps is equal to the number of atomic sites considered [27-29].

\section{Conclusions}

A semi-analytic method for the calculation of the transport properties of Biphenyl molecule in the nearest-neighbor tight-binding approximation have been proposed. This molecule have been studied in two regimes of different coupling strengths with the two semi-infinite leads. Using Green's function techniques within a real-space renormalisation scheme the transmission probability, the current, the thermopower and the DOS have been calculated. We found that transmission presents a peculiar asymmetry in the width of the peaks around the Fermi energy and that the intensity of the current decreases when the coupling decreases. Besides, our results show different transport regimes exhibiting transitions form insulator to semiconductor behaviors. These features give us important insights for considering Biphenyl systems as possible candidates in the design of molecular electronic devices.

\section{Acknowledgments}

We thank to Harald Pleiner (MPI-P, Mainz) for his critical reading and suggestions. J.H.O. acknowledges the financial support from FONDECYT Postdoctoral program fellowship under grant 3130506. D.L. acknowledges partial financial support from FONDECYT 11080229, Basal Program Center for Development of Nanoscience and Nanotechnology (CEDENNA), UTA-project 8750 12. C.A.D. is grateful to the Colombian Agencies CODI-UdeA (Estrategia de Sostenibilidad 20142015 de la UdeA and projects: "Propiedades ópticas de impurezas, excitones y moléculas en puntos 
cuánticos autoensamblados" and "On the way to development of new concept of nanostructure-based THz laser") and El Patrimonio Autónomo Fondo Nacional de Financiamiento para la Ciencia, la Tecnología y la Innovación, Francisco José de Caldas.

\section{Appendix A. Renormalization method and analytical expressions of the Green's functions}

The renormalization of the real system into to an effective 1D dimensional system is through the Dyson equation 5 for the local Green's functions. These functions contain all the information given by the Hamiltonian 1.

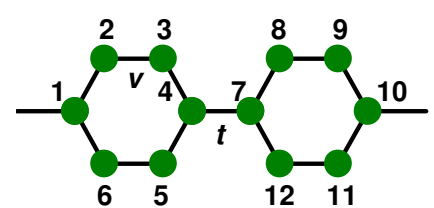

(a) (b)

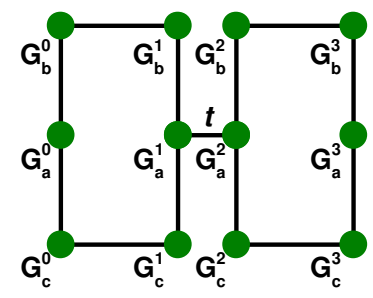

Figure A.7: (Color online) Biphenyl aromatic molecule (a) and geometric pattern of the molecule (b).

In Biphenyl case, the system is determined by twelve carbon atoms. Let us denote the energy as $\epsilon$. We perform the renormalization process of the system without contacts as it is shown Figure A.7. Here each site has an associated atomic Green function undisturbed.

Using the Dyson equation we write the equations of motion of the electron at each site with the respective interaction of their first neighbors. Then the first set of equations for the sites with subscript zero are given by:

$$
\begin{aligned}
G_{00}^{a} & =G_{a}^{0}+G_{a}^{0} v G_{00}^{b}+G_{a}^{0} v G_{00}^{c}, \\
G_{00}^{b} & =G_{b}^{0}+G_{b}^{0} v G_{00}^{a}+G_{b}^{0} v G_{01}^{b}, \\
G_{00}^{c} & =G_{c}^{0}+G_{c}^{0} v G_{00}^{a}+G_{c}^{0} v G_{01}^{b},
\end{aligned}
$$

where,

$$
\begin{aligned}
& G_{01}^{b}=G_{b}^{1} v G_{00}^{b}+G_{b}^{1} v G_{01}^{a}, \\
& G_{01}^{c}=G_{c}^{1} v G_{00}^{c}+G_{c}^{1} v G_{01}^{a} .
\end{aligned}
$$

Taking the above equations, we can solve $G_{00}^{b}$ and $G_{00}^{c}$ :

$$
\begin{aligned}
G_{00}^{b} & =\frac{G_{b}^{0}+G_{b}^{0} v G_{00}^{a}}{1-G_{b}^{0} G_{b}^{1} v^{2}}+\frac{G_{b}^{0} G_{b}^{1} v^{2} G_{01}^{a}}{1-G_{b}^{0} G_{b}^{1} v^{2}} \\
G_{00}^{c} & =\frac{G_{c}^{0}+G_{c}^{0} v G_{00}^{a}}{1-G_{c}^{0} G_{c}^{1} v^{2}}+\frac{G_{c}^{0} G_{c}^{1} v^{2} G_{01}^{a}}{1-G_{c}^{0} G_{c}^{1} v^{2}}
\end{aligned}
$$

Now, we replace (A.6) y (A.7) into (A.1), and taking into account that $G_{a}^{0}=G_{b}^{0}=G_{c}^{0}=G_{b}^{1}=$ $G_{c}^{1}=g_{0}$, where, $g_{0}=1 /(E-\epsilon)$ is the local Green's function, we can obtain:

$$
G_{00}^{a}=\tilde{G}_{0}+\tilde{G}_{0} \tilde{v} G_{01}^{a},
$$

where $\tilde{G}_{0}$ and $\tilde{v}$ are new Green function of effective site and coupling effective inter-site respectively and are given by:

$$
\tilde{G}_{0}=\frac{1}{(E-\widetilde{\epsilon})}=\frac{g_{0}-g_{0}^{3} v^{2}+2 g_{0}^{2} v}{1-3 g_{0}^{2} v^{2}},
$$

and

$$
\tilde{v}=\frac{2 g_{0}^{3} v^{3}}{g_{0}-g_{0}^{3} v^{2}+2 g_{0}^{2} v} .
$$

The same way, we build equations for the site with subscript one:

$$
\begin{gathered}
G_{11}^{a}=G_{a}^{1}+G_{a}^{1} v G_{11}^{b}+G_{a}^{1} v G_{11}^{c}+G_{a}^{1} t G_{12}^{a}, \\
G_{11}^{b}=G_{b}^{1}+G_{b}^{1} v G_{11}^{a}+G_{b}^{1} v G_{10}^{b} \\
G_{11}^{c}=G_{c}^{1}+G_{c}^{1} v G_{11}^{a}+G_{c}^{1} v G_{10}^{c}
\end{gathered}
$$

where,

$$
\begin{aligned}
& G_{10}^{b}=G_{b}^{(0)} v G_{11}^{b}+G_{b}^{(0)} v G_{10}^{a} \\
& G_{10}^{c}=G_{c}^{(0)} v G_{11}^{c}+G_{c}^{(0)} v G_{10}^{a} .
\end{aligned}
$$

From the above set of equations we obtain:

$$
G_{11}^{a}=\tilde{G}_{0}+\tilde{G}_{0} \tilde{v} G_{10}^{a}+\tilde{G}_{0} \tilde{t} G_{12}^{a},
$$

where the effective torsion energy is:

$$
\tilde{t}=\frac{t\left(1-g_{0}^{2} v^{2}\right)}{1-g_{0}^{2} v^{2}+2 g_{0} v}=\frac{v\left(1-g_{0}^{2} v^{2}\right) \cos ^{2}(\varphi)}{1-g_{0}^{2} v^{2}+2 g_{0} v}
$$

with $t=v \cos ^{2}(\varphi)$. For sites 3 and 4 we proceed in the same way. 
Once obtained the Green's functions and coupling effectives to the new dimensional system, we determine analytically the Green's functions $G_{1 N}^{r}$ which can be determined by using equation 5 . With the same procedure used above and adding the molecule-electrodes interactions as follow:

$$
\begin{aligned}
& G_{1 N}^{r}=G_{0}^{1 N}+G_{0}^{11} \sum_{\nu} \Gamma_{R}^{\nu} G_{R N}^{\nu}+G_{0}^{1 N} \sum_{\nu} \Gamma_{L}^{\nu} G_{L N}^{\nu} \\
& G_{N N}^{r}=G_{0}^{N N}+G_{0}^{N 1} \sum_{\nu} \Gamma_{R}^{\nu} G_{R N}^{\nu}+G_{0}^{N N} \sum_{\nu} \Gamma_{L}^{\nu} G_{L N}^{\nu}
\end{aligned}
$$

where $G_{R N}^{\nu}$ and $G_{L N}^{\nu}$ are the Green's functions for the electron from each one of electrodes to the $N-t h$ molecular site. This Green's functions are given by:

$$
\begin{gathered}
G_{R N}^{\nu}=G_{0(R)}^{\nu} \Gamma_{R}^{\nu} G_{1 N}^{r} \\
G_{L N}^{\nu}=G_{0(L)}^{\nu} \Gamma_{L}^{\nu} G_{N N}^{r} .
\end{gathered}
$$

Done the mapping along of the effective molecule Fig. 2, we can write the Green's function $G_{1 N}^{r}$ as:

$$
G_{1 N}^{r}=\frac{G_{1 N}^{0}}{\left(1+i G_{N N}^{0} \frac{\Gamma}{2}\right)^{2}+\left(G_{1 N}^{0}\right)^{2} \frac{\Gamma^{2}}{4}} .
$$

The functions $\left\{G_{1 N}^{0}, G_{N N}^{0}\right\}$ can be obtained with the same method, and they are explicitly given by:

$$
G_{1 N}^{0}=\frac{\tilde{G}_{0}^{4} \tilde{v}^{2} \tilde{t}}{1-2 \tilde{G}_{0}^{2} \tilde{v}^{2}-\tilde{G}_{0}^{2} \tilde{t}^{2}+\tilde{G}_{0}^{4} \tilde{v}^{4}},
$$

and

$$
G_{N N}^{0}=\frac{\tilde{G}_{0}\left(1-\tilde{G}_{0}^{2} \tilde{t}^{2}-\tilde{G}_{0}^{2} \tilde{v}^{2}\right)}{1-2 \tilde{G}_{0}^{2} \tilde{v}^{2}-\tilde{G}_{0}^{2} \tilde{t}^{2}+\tilde{G}_{0}^{4} \tilde{v}^{4}},
$$

where $\Sigma^{L}=\Sigma^{R}=-i \Gamma / 2$. In addition we also need the diagonal Green's functions. For symmetry $G_{22}^{0}=G_{33}^{0}$. Using equation 5 we obtain that:

$$
G_{22}^{0}=\frac{\tilde{G}_{0}\left(1-\tilde{G}_{0}^{2} \tilde{v}^{2}\right)}{1-2 \tilde{G}_{0}^{2} \tilde{v}^{2}-\tilde{G}_{0}^{2} \tilde{t}^{2}+\tilde{G}_{0}^{4} \tilde{v}^{4}} .
$$

These functions contain all the relevant information to compute the quantum transport properties.
[1] A. Lorenzoni, M. Muccini, and F. Mercuri, RSC Adv. 5, 11797 (2015).

[2] P. M. Solomon and N. D. Lang, ACS nano 2, 435 (2008).

[3] E. Leary, A. La Rosa, M. T. González, G. RubioBollinger, N. Agraï, and N. Martín, Chem. Soc. Rev. 44, 920 (2015).

[4] W. Li, K. H. Hendriks, A. Furlan, A. Zhang, M. M. Wienk and R. A. J. Janssen, Chem. Commun. 51, 4290. (2015).

[5] S. K. Maiti, I. J. Nanosc. 6, 415 (2007).

[6] M. Bürkle, J. K. Viljas, D. Vonlanthen, A. Mishchenko, G. Schön, M. Mayor, T. Wand-lowski, and F. Pauly, Phys. Rev. B 85, 075417 (2012).

[7] Y. Xue and M. A. Ratner, Phys. Rev. B 69, 085403 (2004).

[8] L. Malysheva and A. Onipko, Phys. Status Solidi (b) 248, 2676 (2011).

[9] S. K. Maiti, European Phys. J. B 86, 296 (2013).

[10] J. H. Ojeda, R. Rey-González, and D. Laroze, J. App. Phys. 114, 213702 (2013).

[11] J. H. Ojeda, P. Orellana, and D. Laroze, J. Chem. Phys. 140, 104308 (2014).

[12] R. Landauer, IBM Journal of Research and Development 1, 223 (1957).

[13] J. H. Ojeda, M. Pacheco, L. Rosales, and P. A. Orellana, Org. Elec. 13, 1420 (2012).

[14] S. Datta, Quantum transport: atom to transistor (Cambridge University Press, 2005).

[15] J. P. Bergfield, M. A. Solis, and C. A. Stafford, ACS nano 4, 5314 (2010).

[16] Y. Dubi, J. Chem. Phys.138, 114706 (2013).

[17] P. Butcher, Journal of Physics: Cond. Matter. 2, 4869 (1990).

[18] L. Venkataraman, J. E. Klare, C. Nuckolls, M. S.Hybertsen, and M. L. Steigerwald, Nature 442, 904 (2006).

[19] J. H. Ojeda, C. A. Duque, and D. Laroze, Phys. E, 62, 15 (2014).

[20] G. C. Solomon, C. Herrmann, T. Hansen, V. Mujica and M. A. Ratner, Nature Chemistry, 2, 223 (2010).

[21] T. Markussen, Robert Stadler, and K. S. Thygese, Nano Lett. , 10, 4260 (2010).

[22] H. Vazquez, R. Skouta, S. Schneebeli, M. Kamenetska, R. Breslow, L. Venkataraman and M.S. Hybertsen, Nature Nanotechnology, 7, 663 (2012).

[23] M. Paulsson, S. Datta, Phys. Rev. B 67, 241403 (2003).

[24] Y. Xue and M. A. Ratner, Phys. Rev. B 68, 115406 (2003).

[25] K. H. Khoo, Y. Chen, S. Li, and S. Y. Quek, Phys. Chem. Phys. 17, 77 (2015).

[26] S. Y. Quek, H. J. Choi, S. G. Louie, and J. B. Neaton, Nano Lett. 9, 3949 (2009).

[27] J. Wang, S. Tang, A. Sandström, and L. Edman, ACS Appl. Mater. Interfaces 7, 2784 (2015). 
[28] T. Kirchner, B. Briechle, E. Scheer, J. Wolf, T. Huhn, and A. Erbe, Acta Phys. Pol. 121, 410 (2012).

[29] X.-W. Yan, R.-J. Liu, Z.-L. Li, B. Zou, X.-N. Song, and C.-K. Wang, Chem. Phys. Lett. 429, 225 (2006). 